\section{Developing new automated alternation flicker using optic disc photography for the detection of glaucoma progression}

J Ahn' ${ }^{1}$ IS Yun ${ }^{1}$, HG Yoo', J-J Choi' ${ }^{2}$ and M Lee ${ }^{3}$

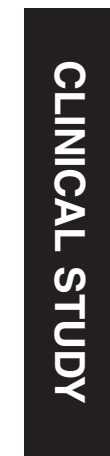

\begin{abstract}
Purpose To evaluate a progression-detecting algorithm for a new automated matched alternation flicker (AMAF) in glaucoma patients.
\end{abstract}

Methods Open-angle glaucoma patients with a baseline mean deviation of visual field (VF) test $>-6 \mathrm{~dB}$ were included in this longitudinal and retrospective study. Functional progression was detected by two VF progression criteria and structural progression by both AMAF and conventional comparison methods using optic disc and retinal nerve fiber layer (RNFL) photography. Progression-detecting performances of AMAF and the conventional method were evaluated by an agreement between functional and structural progression criteria. RNFL thickness changes measured by optical coherence tomography (OCT) were compared between progressing and stable eyes determined by each method.

Results Among 103 eyes, 47 (45.6\%), $21(20.4 \%)$, and $32(31.1 \%)$ eyes were evaluated as glaucoma progression using AMAF, the conventional method, and guided progression analysis (GPA) of the VF test, respectively. The AMAF showed better agreement than the conventional method, using GPA of the VF test $(\kappa=0.337 ; P<0.001$ and $\kappa=0.124 ; P=0.191$, respectively). The rates of RNFL thickness decay using OCT were significantly different between the progressing and stable eyes when progression was determined by AMAF $(-3.49 \pm 2.86 \mu \mathrm{m}$ per year $v s-1.83 \pm 3.22 \mu \mathrm{m}$ per year;

$P=0.007)$ but not by the conventional method $(-3.24 \pm 2.42 \mu \mathrm{m}$ per year $v s-2.42 \pm 3.33 \mu \mathrm{m}$ per year; $P=0.290$ ).

Conclusions The AMAF was better than the conventional comparison method in discriminating structural changes during glaucoma progression, and showed a moderate agreement with functional progression criteria.

Eye (2017) 31, 119-126; doi:10.1038/eye.2016.204; published online 23 September 2016

Introduction

Glaucoma is an irreversible optic neuropathy resulting from retinal ganglion cell death that leads to progressive visual field (VF) loss. Glaucomatous damage is characterized by structural changes of the optic nerve head $(\mathrm{ONH})$ and retinal nerve fiber layer (RNFL), functional changes of the VF, and ultimate loss of central visual acuity. Structural changes may precede the development of the functional VF defects. ${ }^{1,2}$ Early detection of these changes around the $\mathrm{ONH}$ is therefore essential in diagnosing and monitoring glaucoma. In glaucoma clinics, optical coherence tomography (OCT) and ONH photography (with red-free RNFL photography) have been commonly used to detect structural deterioration associated with glaucoma.

New and more sensitive imaging devices to detect structural progression have been recently developed. Guided progression analysis (GPA) using Cirrus OCT (Carl Zeiss Meditec) is an example of such a device that detects structural changes of RNFL using statistical methods. ${ }^{3,4}$ As technology advances, more advanced OCT devices have been developed and are rapidly replacing previous models. For glaucoma progression analysis, it is difficult to compare OCT data directly if we compare the data from different OCT machines. 5,6 Therefore, careful analyses of sequential optic disc photographs are still useful to determine long-term changes
${ }^{1}$ Department of Ophthalmology, Ajou University School of Medicine, Suwon, Korea

${ }^{2}$ Department of Digital Media, Ajou University, Suwon, Korea

${ }^{3}$ Department of Ophthalmology, DMC Bundang Jesaeng General Hospital, Seongnam, Korea

Correspondence:

M Lee, Department of Ophthalmology, Bundang Jesaeng General Hospital, 20, Seohyeon-ro 180 beon-gil, Bundaung-gu 463-774, Sungnam-si, Korea Tel: +82 31779 0297; Fax: +82 317790896 . E-mail: mvdevil@naver.com

Received: 10 February 2016 Accepted in revised form: 17 August 2016 Published online: 23 September 2016 
of ONH and RNFL defects in glaucoma. To improve the detection ability of early $\mathrm{ONH}$ structural changes, a novel method using alternation flicker chronoscopy has been introduced. Alternating flickers can be achieved by manually aligning and alternating images of fundus photographs taken sequentially with overlapping slide projectors or by manual registration of digital images. ${ }^{7}$ This technique has been reported to be effective and comparable to photographic review in several studies. ${ }^{8-10}$ Automated flicker has recently become available, rendering it possible to make automated flicker images using Matched-Flicker 8 software (ver. 1.2; EyeIC, Narberth, PA, USA). This approach has an excellent ability to detect $\mathrm{ONH}$ contour changes, parapapillary atrophy, and disc hemorrhages. ${ }^{7,11,12}$

We have developed a new algorithm, by collaborating with the engineering department, to make automated flickering images of ONH and RNFL photography for clinical purposes. To make matched alternation flicker images using two fundus photographs taken at different time points using different camera angles, each image needs to be processed to adjust the size and rotation angle. In addition, detection of changes in RNFL defects can be a problem if two fundus images have different brightnesses. An ideal algorithm should detect changes that are not associated with the image-capturing procedure, but with glaucoma progression itself. In the following report, we investigated the reproducibility of our algorithm for automatically matched alternation flicker (AMAF), and compared its ability to detect glaucoma progression with the conventional method using serial ONH and RNFL photographs.

\section{Materials and methods}

\section{Selection of patients}

In this longitudinal and retrospective study, the medical records of patients who visited the glaucoma clinic from January 2010 to December 2012 at the Department of Ophthalmology, Ajou University Hospital, for glaucoma evaluation, were reviewed. The study adhered to the Declaration of Helsinki and was approved by the Institutional Review Board of Ajou University Hospital (AJIRB-MED-MDB-13-137).

Primary open-angle glaucoma (POAG) patients with baseline mean deviation (MD) values better than $-6 \mathrm{~dB}$ were identified from the medical records. Baseline and last fundus photography examinations had an interval of $\geq 1$ year. All patients received at least five VF tests for GPA using the Humphrey Field Analyzer (HFA; Carl Zeiss Meditec, Dublin, CA, USA). All patients underwent a comprehensive ophthalmic examination, including a medical history review, best-corrected visual acuity, spherical equivalent of the refractive error, slit lamp biomicroscopy, central corneal thickness (DGH-500; DGH Technology, Exton, PA, USA), intraocular pressure (IOP) measurement using Goldman applanation tonometry, gonioscopy, dilated fundus examination, and automated perimetry (HFA) using the Swedish interactive thresholding algorithm with standard 24-2 strategies. Stereo optic-disc photography (ODP; AFC-210; NIDEK, Aichi, Japan) and time domain OCT (Stratus OCT; Carl Zeiss Meditec) were performed on the same day. VF tests, and ODP and OCT examinations, were performed within 3 months of other ocular examinations. All the patients in this study had an IOP $<22 \mathrm{~mm} \mathrm{Hg}$ at every visit with or without medication, and had gonioscopic open-angle glaucoma without secondary causes. The baseline VF tests showed at least two reproducible VF defects compatible with glaucoma according to Anderson's criteria for minimal abnormality in glaucoma. ${ }^{13}$ RNFL defects, corresponding with VF defects, were noted in red-free RNFL photography and/or OCT.

\section{Creating the application}

This application was designed to help ophthalmologists detect differences between two fundus images taken at two different time points, by alternately exposing the images. Owing to the time gap between the two images, there are usually changes in scale, rotation, translation, brightness, and noise. A well-known computer vision technique, Speeded Up Robust Features (SURF), ${ }^{14}$ was used to better match corresponding features of the two images. SURF facilitated feature correspondence that was scale- and rotation-invariant, and also accounted for noise and photometric deformations. Using the feature correspondence, a transformation matrix was used to deform one image to another by solving a linear least squares optimization problem. The feature points in one image therefore had almost the same positions relative to their corresponding feature points as the other deformed image. Attempts to match all the parts in an image sometimes caused unwanted distortion of the image, especially around the $\mathrm{ONH}$. To increase the degree of match around the $\mathrm{ONH}$, we therefore added a function that allowed the examiners to specify an area as a reference for image fusion (Figure 1). After this feature matching, the brightness levels of the two matched images were adjusted as closely as possible. This application provided users with controls for the scale, rotation invariants, and exposure frequency. The application was implemented using the $\mathrm{C}++$ programming language, OpenCV, and Fast Light Toolkit libraries using the Windows 7 operating system (Microsoft Corp., Redmond, WA, USA). 


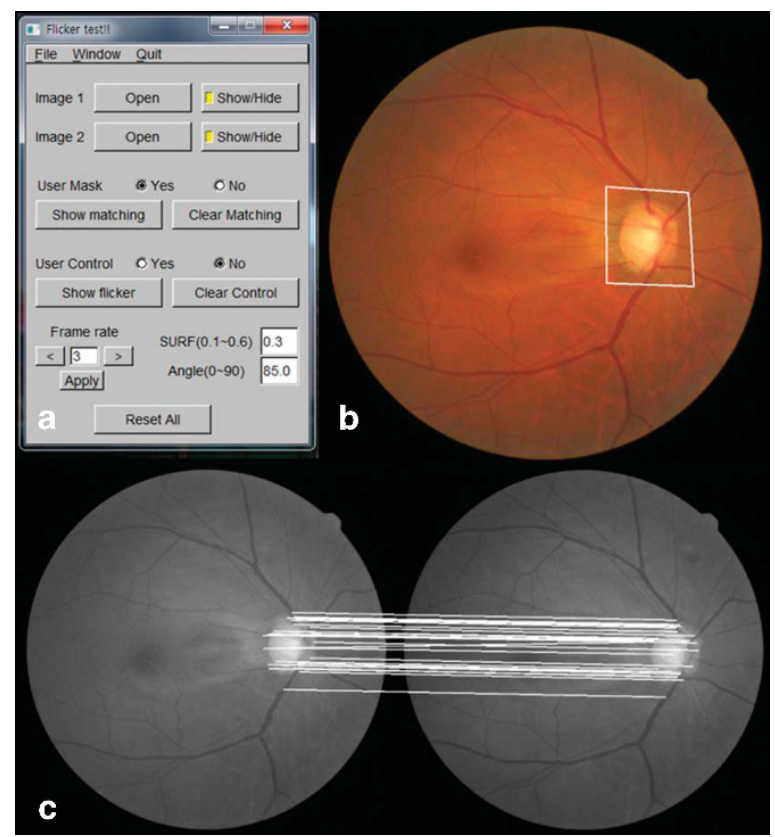

Figure 1 (a) The appearance of the application. (b) When examiner check 'User mask' to 'Yes' on flicker applications, examiner can mark area that examiners want to make it a reference area for image fusion. (c) Result of matching point after examiner marks area that examiners want to make it a reference area.

We conducted a separate study in a different set of subjects (66 eyes of 66 patients), prior to this study to evaluate agreements among three examiners who identified glaucomatous structural changes using AMAF method. In that study, intraclass correlation (ICC) coefficient were from 0.777 to 0.850 for inter-reader agreement and from 0.853 to 0.988 for intra-reader agreement. $^{15}$

\section{Making flicker images and the determination of progression}

Two original fundus photographs taken at two different time points for each patient were extracted from the fundus photography database. Using our flicker application for AMAF, the first and second images were selected and three hertz flicker images were automatically created with contrast adjustments. If the examiner determined that the generated flicker images were sufficient to determine fundus changes, the glaucoma progression was evaluated by a single glaucoma specialist (ML) using a 3-point grading scale. Grade 3 was defined as definite glaucomatous change in the fundus images, and grade 2 was defined as a fundus change with suspected glaucoma. We only used the 3-point grading scale to evaluate glaucoma progression. If there was no detectable change in the flicker image, it was designated as grade 1 . To compare the progression-detecting ability of AMAF with the parallel comparisons, glaucoma progression was evaluated in terms of four different $\mathrm{ONH}$ and RNFL changes, involving changes in the neuroretinal rim of the optic disc, in the path of the vessel around the optic disc, and in the widening and deepening of the RNFL defect (defined as a loss or decrease in RNFL striation leading to darkening of the previous defects).

Conventional side-by-side comparisons of fundus photographs were performed by the same examiner (ML) in a blinded manner. Another operator prepared two fundus photographs to make an automated flicker image with randomly assigned serial numbers. The inspector (ML) evaluated it in the same manner using the 3-point grading scale.

VF progression was determined independently after evaluating glaucoma progression using AMAF and sideby-side comparisons in a blinded manner. VF progression was defined by GPA (integrated in the HFA) and by the collaborative initial glaucoma treatment study (CIGTS) criteria. A 'likely progression' using the GPA software was defined as progression of GPA criterion. An increase in CIGTS score of three or more compared with an average of two baseline VF tests was defined as progression of CIGTS criterion. ${ }^{16}$ A VF test conducted on the same day or within 3 months from the first fundus photography was used as a baseline VF. A VF test conducted at the closest time point (within 3 months) of the final fundus photography was used to determine glaucoma progression.

For progressing and stable eyes assessed by both AMAF and conventional ODP analyses, changes of average RNFL thickness using Stratus OCT were compared between the two methods.

\section{Statistical analysis}

Descriptive results were expressed as means $\pm S D$, and $P$-values $<0.05$ were considered statistically significant. The kappa statistic was used to quantify and evaluate the agreement between evaluations of glaucoma progression determined by different methods. A kappa value of $0.1-0.2$ was considered as slight agreement, $0.21-0.40$ as moderate agreement, $0.41-0.60$ as fair agreement, $0.61-0.80$ as substantial agreement, and $0.81 \sim 0.99$ as almost perfect agreement. ${ }^{17}$ SPSS (ver. 20.0; SPSS Inc., Chicago, IL, USA) and MedCalc software (ver. 12.0; Mariakerke, Belgium) were used for analyses.

\section{Results}

In total, 103 POAG patients were enrolled after screening 210 patients and excluding patients who did not meet the inclusion criteria. The mean age of the patients was 
$52.8 \pm 9.9$ years (range: $19-79$ years), and 54 patients $(52.4 \%)$ were male. The mean interval between the first baseline fundus photography and the final examination was $28.7 \pm 9.0$ months. The MD values decreased significantly from $-0.93 \pm 2.14 \mathrm{~dB}$ to $-0.62 \pm 2.93 \mathrm{~dB}$, and both pattern SD values and CIGTS scores significantly increased. RNFL thicknesses also decreased significantly in all quadrants, including the average value (Table 1).

Kappa coefficients showed almost perfect intraobserver agreement $(\kappa=0.863,95 \% \mathrm{CI}=0.806-0.919, P<0.001)$ using AMAF analysis and substantial agreement $(\kappa=0.797,95 \% \mathrm{CI}=0.716-0.878, P<0.001)$ using conventional side-by-side comparisons of ODP.

We compared two methods for assessing structural progression based on the agreement with two progression criteria of functional tests using kappa statistics.

The AMAF showed moderate agreement with both GPA $(\kappa=0.337)$ and CIGTS $(\kappa=0.241)$ progression criteria, whereas the conventional comparison method showed no significant agreement with two criteria for functional progression (Table 2). The AMAF and conventional comparison showed a fair agreement with each other $(\kappa=0.468, P<0.001)$.

The AMAF designated 47 of 103 eyes with glaucoma progression, whereas 21 eyes were designated with glaucoma progression using the conventional comparison method. The two methods showed a substantial agreement in detecting widening of RNFL defects $(\kappa=0.785 ; P<0.001)$. RNFL defect depth changes $(\kappa=0.354 ; P<0.001)$ and changes of the neuroretinal $\operatorname{rim}(\kappa=0.241 ; P=0.001)$ showed moderate agreement. Detection of glaucoma progression using a change in path of the vessel around the ONH showed slight agreement $(\kappa=0.109 ; P=0.015)$. AMAF detected changes of the vessel around the optic disc in 15 eyes, but only a single eye was detected with a vessel change using the conventional comparison method (Table 3).

The rate of average RNFL thickness decrease of progressing eyes $(-3.49 \pm 2.86 \mu$ m per year $)$ was significantly different from that of stable eyes $(-1.83 \pm 3.22 \mu \mathrm{m}$ per year; $P=0.007)$ when progression was determined using AMAF. However, there was no

Table 1 Descriptive statistics of enrolled subjects (means \pm SD)

\begin{tabular}{lcrl}
\hline & Baseline examination & Last examination & P-value \\
\hline Duration & & $28.7 \pm 9.0$ months & $<0.001$ \\
MD, dB & $-0.93 \pm 2.14$ & $-1.62 \pm 2.93$ & $<0.001$ \\
PSD, dB & $3.97 \pm 2.79$ & $4.52 \pm 3.41$ & $<0.001$ \\
CIGTS score & $2.06 \pm 2.43$ & $2.78 \pm 2.93$ & $<0.001$ \\
& & & $<0.001$ \\
RNFL thickness measured by OCT $(\mu \mathrm{m})$ & & & $<0.001$ \\
Average & $87.79 \pm 12.78$ & $81.86 \pm 13.79$ & $<0.001$ \\
Temporal quadrant & $69.84 \pm 13.81$ & $65.85 \pm 13.15$ & $<0.001$ \\
Superior quadrant & $107.38 \pm 20.35$ & $100.64 \pm 22.19$ & $68.17 \pm 15.00$ \\
Nasal quadrant & $71.83 \pm 15.16$ & $92.82 \pm 25.15$ & $<$ \\
Inferior quadrant & $101.02 \pm 25.38$ & & $<$ \\
\hline
\end{tabular}

Abbreviations: CIGTS, collaborative initial glaucoma treatment study; MD, mean deviation; OCT, optical coherence tomography; PSD, pattern SD; RNFL, retinal nerve fiber layer.

Statistical significance was evaluated by paired $T$-test.

Table 2 Agreement between image analysis methods and VF criteria to detect glaucoma progression

\begin{tabular}{|c|c|c|c|c|c|c|}
\hline & \multicolumn{3}{|c|}{ GPA progression criteria } & \multicolumn{3}{|c|}{ CIGTS progression criteria } \\
\hline & Stable & Progress & к (P-value) & Stable & Progress & $\kappa(\mathrm{P}$-value $)$ \\
\hline \multicolumn{7}{|l|}{$A M A F$} \\
\hline Stable & 47 & 9 & $0.337(<0.001)$ & 51 & 5 & $0.241(0.003)$ \\
\hline Progress & 24 & 23 & & 32 & 15 & \\
\hline \multicolumn{7}{|c|}{ Conventional methods } \\
\hline Stable & 59 & 23 & $0.124(0.191)$ & 69 & 13 & $0.178(0.071)$ \\
\hline Progress & 12 & 9 & & 14 & 7 & \\
\hline
\end{tabular}

Abbreviations: AMAF, automated matched alternating flicker; CIGTS, collaborative initial glaucoma treatment study; GPA, glaucoma progression analysis; $\kappa$, Kappa coefficient. 
Table 3 Agreement between matched flicker and conventional comparison method to detect changes of fundus photography

\begin{tabular}{|c|c|c|c|c|c|c|}
\hline & & & \multicolumn{3}{|c|}{ Conventional comparison method } & \multirow[t]{2}{*}{ P-value } \\
\hline & & & Stable & Progress & Kappa & \\
\hline \multirow[t]{2}{*}{ RNFL defect widening } & \multirow[t]{2}{*}{ AMAF } & Stable & 75 & 0 & \multirow[t]{2}{*}{0.785} & \multirow[t]{2}{*}{$<0.001$} \\
\hline & & Progress & 8 & 20 & & \\
\hline \multirow[t]{2}{*}{ RNFL defect depth deepening } & \multirow[t]{2}{*}{ AMAF } & Stable & 74 & 0 & \multirow[t]{2}{*}{0.354} & \multirow[t]{2}{*}{$<0.001$} \\
\hline & & Progress & 21 & 8 & & \\
\hline \multirow[t]{2}{*}{ Rim contour change } & \multirow[t]{2}{*}{ AMAF } & Stable & 76 & 1 & \multirow[t]{2}{*}{0.241} & \multirow[t]{2}{*}{0.001} \\
\hline & & Progress & 21 & 5 & & \\
\hline \multirow[t]{2}{*}{ Vessel contour change } & \multirow[t]{2}{*}{ AMAF } & Stable & 88 & 0 & \multirow[t]{2}{*}{0.109} & \multirow[t]{2}{*}{0.015} \\
\hline & & Progress & 14 & 1 & & \\
\hline
\end{tabular}

Abbreviations: AMAF, automated matched alternating flicker; RNFL, retinal nerve fiber layer.

Table 4 Comparison of retinal nerve fiber layer decay between progressing and stable eyes according to the progression criteria

\begin{tabular}{lccr}
\hline Progression criteria & \multicolumn{2}{c}{ R-value $^{\text {R }}$} \\
\cline { 2 - 3 } & Progressing eyes & Stable eyes & \\
\hline Conventional methods & $-3.24 \pm 2.42$ & $-2.42 \pm 3.33$ & 0.290 \\
AMAF & $-3.49 \pm 2.86$ & $-1.83 \pm 3.22$ & 0.007 \\
GPA & $-3.81 \pm 3.20$ & $-2.87 \pm 3.86$ & 0.008 \\
CIGTS & $-3.63 \pm 2.24$ & $-2.34 \pm 3.31$ & 0.101 \\
Conventional methods combined with GPA & $-3.56 \pm 2.98$ & $-1.86 \pm 3.12$ & 0.006 \\
AMAF combined with GPA & $-3.52 \pm 3.02$ & $-1.47 \pm 2.99$ & 0.001 \\
\hline
\end{tabular}

Abbreviations: AMAF, automated matched alternating flicker; CIGTS, collaborative initial glaucoma treatment study; GPA, glaucoma progression analysis; RNFL, retinal nerve fiber layer. ${ }^{a}$ Independent student $t$-test between progressing and stable eyes.

significant difference between progressing and stable eyes using the conventional method $(-3.24 \pm 2.42$ vs $-2.42 \pm 3.33 \mu \mathrm{m}$ per year; $P=0.290$ ). The average RNFL thickness of progressing eyes decreased significantly more than that of stable eyes when the GPA criterion was used $(-3.81 \pm 3.20$ vs $-2.87 \pm 3.86 \mu \mathrm{m}$ per year; $P=0.008)$, whereas the rate of RNFL decrease between progressing and stable eyes was not significantly different when the CIGTS criterion was used $(-3.63 \pm 2.24$ vs $-2.34 \pm 3.31 \mu \mathrm{m}$ per year; $P=0.101)$.

When progression was determined by GPA combined with AMAF or the conventional method, the average RNFL thickness of progressing eyes decreased significantly more than that of stable eyes using both the AMAF+GPA criteria $(-3.52 \pm 3.02 v s-1.47 \pm 2.99 \mu \mathrm{m}$ per year; $P=0.001)$ and the conventional comparison + GPA criteria. $(-3.56 \pm 2.98$ vs $-1.86 \pm 3.12 \mu \mathrm{m}$ per year; $P=0.006$; Table 4).

\section{Discussion}

Previous studies have reported that alteration flicker chronoscopy is more advantageous in detecting changes of the optic disc and peripapillary structures than conventional side-by-side comparison of fundus photographs. $7,9,18,19$ Automated matched flicker was developed using evolving imaging technology. It can better match the flicker images using simple operations, and has been reported to have good inter-reader and intra-reader agreements. ${ }^{10}$ It has also been reported that flicker chronoscopy was better than the conventional side-by-side comparison method for evaluating the progression of glaucoma, and was better at detecting changes in the contour of the $\mathrm{ONH}$, detecting increases in the area of the peripapillary atrophy, and detecting the appearance and disappearance of disc hemorrhages. ${ }^{7,11,12,20}$

Using AMAF to detect structural changes during glaucoma progression, we focused on changes in the shape of the neuroretinal rim of the $\mathrm{ONH}$, changes of the vessel contour around the optic disc, widening of the RNFL defects, and deepening (defined as loss or decrease in RNFL striation leading to darkening of the previous defects even after adjusting brightness and contrast of images compared) of RNFL defects. Development of disc hemorrhages and peripapillary atrophy changes were not included in the progression criteria if there were no other associated changes. To enhance the ability to detect 
glaucomatous changes, we have made an application to adjust one image to another based on the optic disc margin and major vessels and each points around the optic nerve head were automatically matched to minimize image distortion around optic nerve even though it might increase the distortion of far periphery of the fundus image. Although the matching process is automated using AMAF, an examiner can directly specify the desired matching area using stable structural markers, including the optic disc margin and major blood vessels. The AMAF application can automatically adjust the brightness of the two fundus photographs to increase the brightness of the darker image before performing the automated matched flicker. And with our application examiners can increase or decrease the sensitivity of the founded intersection points easily and can adjust matched flicker images such that the desired degree of fusion. In addition, when we look at the flicker pictures continuously, it is difficult to know whether any photos are firstly recorded. To overcome this disadvantage AMAF automatically marks sequence of recorded images. And to improve the convenience of reading, frequency of flickering of images also can be adjusted easily before and after making automated flicker. These new features of AMAF improved the agreement between structural and functional progression criteria in our study (Table 2; Supplementary Video).

The agreement between structural and functional progression criteria and the ability to detect glaucoma progression are known to be influenced by both detection methods and the severity of glaucoma. ${ }^{21,22}$ In a recent observational cohort study, progression of glaucoma was detected by stereo photographs, GPA of the HFA, and GPA using the Cirrus spectral domain OCT in 6.9\%, 15\%, and $25.6 \%$ of eyes, respectively. In this study, normal, suspected glaucoma, and glaucoma patients were included in 246 eyes of 148 patients. ${ }^{23}$ The detection rate of stereo photography was lower than that of the VF test in those patients with early stages of glaucoma. A higher detection rate of glaucoma progression was reported using flicker chronoscopy in another study, and 50 (48.5\%) of 103 eyes showed at least one sign of structural progression, although the severity of glaucoma was not reported. ${ }^{24}$ In our study, the conventional comparison method, AMAF, and GPA of the VF test could detect glaucoma progression in $20.4 \%, 45.6 \%$, and $31.1 \%$ of the eyes with a mild baseline VF defect $(\mathrm{MD}>-6 \mathrm{~dB})$, respectively. Consistent with a previous study, ${ }^{23}$ we also found that stereo photographs detected fewer eyes with glaucoma progression than GPA of the VF test in the early stages of glaucoma, but AMAF could detect progression in more eyes than GPA. These reports are comparable with previous studies using flicker chronoscopy. ${ }^{24}$ In a previous study, ${ }^{23}$ the agreement between stereo photographs and GPA of the VF test was moderate $(\kappa=0.21)$, as was the agreement between the AMAF $(\kappa=0.337)$ and the conventional method $(\kappa=0.124)$ in our study (Table 2). Compared with the conventional comparison method, AMAF improved agreement with the functional test and performance. It identified structural changes caused by glaucoma progression because it probably detected subtle changes of the RNFL, neuroretinal rim, and blood vessel contours (Table 3), and produced more reproducible results.

Structural progression was determined by a single grader in our study, although each image for grading was prepared by another examiner in a masked manner. To overcome any discrepancies, we compared the RNFL thickness decay of progressing eyes with that of stable eyes in each progression criteria, because the rate of structural progression should be faster in progressing eyes than in stable ones if each criterion identified the true progression caused by glaucoma (Table 4). On the basis of the limited number of OCT examinations, the RNFL thickness decay was not calculated from linear regression analysis, but from the difference between the first and the last examinations during the follow-up periods. However, the rate of average RNFL thickness decrease in our study was comparable to a previous report using the same OCT (between -2.22 and $-7.60 \mu \mathrm{m}$ per year for the Stratus OCT; Leung et $a l^{25}$ ). In our series, AMAF and GPA showed greater RNFL thickness decreases in progressing eyes than stable eyes, whereas the conventional comparison method and the CIGTS did not. It is possible that the flicker technology and GPA criteria outperformed the conventional comparison and CIGTS methods in detecting glaucoma progression in patients with mild baseline VF defects. Both progressing and stable eyes, by all criteria, showed significant RNFL thickness decreases from the baseline results in our study. Liu et $a^{26}$ reported that RNFL thicknesses decreased significantly even in eyes showing no progression, when estimated by conventional methods using both GPA of the VF test and stereo disc photography. Although the rate of RNFL decay of the study of Liu et $a^{26}$ (between $-0.71 \pm 0.09$ and $-1.0 \pm 0.20 \mu \mathrm{m}$ per year) was less than that of our results $(-1.83 \pm 3.22$ and $-2.87 \pm 3.86 \mu \mathrm{m}$ per year in stable eyes using AMAF and GPA criteria, respectively), the use of different devices (Stratus vs Cirrus) and decaycalculating algorithms may have influenced the results. The rate of RNFL thickness decrease as reported using Cirrus OCT may be slower than that using Stratus OCT in the same patients. ${ }^{25}$ When GPA criterion was combined with the conventional comparison method, RNFL thickness decreases between progressing and stable eyes were significantly different (Table 4). On the basis of these results, conventional stereo photographs combined with 
GPA can improve the ability to assess glaucoma progression when flicker technology is not available.

Our study had some limitations. First, it used a retrospective design and a limited number of OCT examinations. The number of examinations was therefore insufficient for regression analysis to detect changes of RNFL thicknesses. Futhermorer, the optic disc photographs were interpreted by a single grader, although a masked method was used.

In conclusion, optic disc photography using a flicker chronoscopic method, AMAF, can detect more glaucoma progression than GPA of the VF in patients with a mild baseline VF defect. AMAF was better than the side-byside comparison method in discriminating glaucomatous changes, and showed moderate agreement with functional progression using the VF test.

\section{Summary}

What was known before

- Alteration flicker chronoscopy is more advantageous in detecting changes of the optic disc and peripapillary structures than conventional side-by-side comparison of fundus photographs.

\section{What this study adds}

- Optic disc photography using a flicker chronoscopic method (AMAF) can detect more glaucoma progression than GPA of the visual field (VF) in patients with a mild baseline VF defect.

- AMAF was better than the side-by-side comparison method in discriminating glaucomatous changes, and showed moderate agreement with functional progression using the VF test.

\section{Conflict of interest}

The authors declare no conflict of interest.

\section{Acknowledgements}

This study has been supported by the 2013 Korean Glaucoma Society Research Fund. The English in this document has been checked by at least two professional editors, both native speakers of English. For a certificate, please see: http:/ /www.textcheck.com/certificate/ u8Sjsx.

\section{References}

1 Harwerth RS, Carter-Dawson L, Shen F, Smith 3rd EL, Crawford ML. Ganglion cell losses underlying visual field defects from experimental glaucoma. Invest Ophthalmol Vis Sci 1999; 40(10): 2242-2250.
2 Kass MA, Heuer DK, Higginbotham EJ, Johnson CA, Keltner JL, Miller JP et al. The Ocular Hypertension Treatment Study: a randomized trial determines that topical ocular hypotensive medication delays or prevents the onset of primary open-angle glaucoma. Arch Ophthalmol 2002; 120 (6): 701-713.

3 Alencar LM, Zangwill LM, Weinreb RN, Bowd C, Vizzeri G, Sample PA et al. Agreement for detecting glaucoma progression with the GDx guided progression analysis, automated perimetry, and optic disc photography. Ophthalmology 2010; 117(3): 462-470.

4 Leung CK, Yu M, Weinreb RN, Lai G, Xu G, Lam DS. Retinal nerve fiber layer imaging with spectral-domain optical coherence tomography: patterns of retinal nerve fiber layer progression. Ophthalmology 2012; 119(9): 1858-1866.

5 Molnar A, Holmstrom G, Larsson E. Macular thickness assessed with spectral domain OCT in a population-based study of children: normative data, repeatability and reproducibility and comparison with time domain OCT. Acta Ophthalmol 2015; 93(5): 470-475.

6 Pierro L, Gagliardi M, Iuliano L, Ambrosi A, Bandello F. Retinal nerve fiber layer thickness reproducibility using seven different OCT instruments. Invest Ophthalmol Vis Sci 2012; 53(9): 5912-5920.

7 Berger JW, Patel TR, Shin DS, Piltz JR, Stone RA. Computerized stereochronoscopy and alternation flicker to detect optic nerve head contour change. Ophthalmology 2000; 107(7): 1316-1320.

8 Leske MC, Heijl A, Hyman L, Bengtsson B. Early Manifest Glaucoma Trial: design and baseline data. Ophthalmology 1999; 106(11): 2144-2153.

9 Heijl A, Bengtsson B. Diagnosis of early glaucoma with flicker comparisons of serial disc photographs. Invest Ophthalmol Vis Sci 1989; 30(11): 2376-2384.

10 Cymbor M, Lear L, Mastrine M. Concordance of flicker comparison versus side-by-side comparison in glaucoma. Optometry 2009; 80(8): 437-441.

11 Syed ZA, Radcliffe NM, De Moraes CG, Smith SD, Liebmann JM, Ritch R. Automated alternation flicker for the detection of optic disc haemorrhages. Acta Ophthalmol 2012; 90(7): 645-650.

12 VanderBeek BL, Smith SD, Radcliffe NM. Comparing the detection and agreement of parapapillary atrophy progression using digital optic disk photographs and alternation flicker. Graefes Arch Clin Exp Ophthalmol 2010; 248(9): 1313-1317.

13 Hodapp E, Parrish RK, Anderson DR. Clinical Decisions in Glaucoma. Mosby: St. Louis, MO, USA, 1993.

14 Bay H, Tuytelaars T, Gool LV. SURF: speeded up robust features. Comput Vis Image Und 2008; 110(3): 346-359.

15 Yun I, Rho S, Jang S, Ahn J, Choi J, Lee M. Agreement of new automated matched alternation flicker using undilatedfundus photography for the detection of glaucomatous structural change. Curr Eye Res 2016; e-pub ahead of print 15 July 2016.

16 Musch DC, Gillespie BW, Lichter PR, Niziol LM, Janz NKInvestigators CS. Visual field progression in the Collaborative Initial Glaucoma Treatment Study the impact of treatment and other baseline factors. Ophthalmology 2009; 116(2): 200-207.

17 Landis JR, Koch GG. The measurement of observer agreement for categorical data. Biometrics 1977; 33(1): 159-174.

18 Radcliffe NM, Sehi M, Wallace IB, Greenfield DS, Krupin T, Ritch R. Comparison of stereo disc photographs and 
alternation flicker using a novel matching technology for detecting glaucoma progression. Ophthalmic Surg Lasers Imaging 2010; 41(6): 629-634.

19 Bengtsson B, Krakau CE. Flicker comparison of fundus photographs. A technical note. Acta Ophthalmol (Copenh) 1979; 57(3): 503-506.

20 Syed ZA, Radcliffe NM, De Moraes CG, Liebmann JM, Ritch R Detection of progressive glaucomatous optic neuropathy using automated alternation flicker with stereophotography. Arch Ophthalmol 2011; 129(4): 521-522.

21 Malik R, Swanson WH, Garway-Heath DF. 'Structurefunction relationship' in glaucoma: past thinking and current concepts. Clin Experiment Ophthalmol 2012; 40(4): 369-380.

22 Harwerth RS, Wheat JL, Fredette MJ, Anderson DR. Linking structure and function in glaucoma. Prog Retin Eye Res 2010; 29(4): 249-271.

23 Banegas SA, Anton A, Morilla-Grasa A, Bogado M, Ayala EM, Moreno-Montanes J. Agreement among spectral-domain optical coherence tomography, standard automated perimetry, and stereophotography in the detection of glaucoma progression. Invest Ophthalmol Vis Sci 2015; 56(2): 1253-1260.

24 Chee RI, Silva FQ, Ehrlich JR, Radcliffe NM. Agreement of flicker chronoscopy for structural glaucomatous progression detection and factors associated with progression. Am J Ophthalmol 2013; 155(6): 983-990 e981.

25 Leung CK, Chiu V, Weinreb RN, Liu S, Ye C, Yu M et al. Evaluation of retinal nerve fiber layer progression in glaucoma: a comparison between spectral-domain and time-domain optical coherence tomography. Ophthalmology 2011; 118(8): 1558-1562.

26 Liu T, Tatham AJ, Gracitelli CP, Zangwill LM, Weinreb RN, Medeiros FA. Rates of retinal nerve fiber layer loss in contralateral eyes of glaucoma patients with unilateral progression by conventional methods. Ophthalmology 2015; 122(11): 2243-2251.

Supplementary Information accompanies this paper on Eye website (http://www.nature.com/eye) 